the models developed by Hall and colleagues (2011) and data from the 1990 Canadian Health Promotion Survey.

Results Our analysis identifies a 95.8 kilocalorie per capita per day increase in caloric intake after CUSFTA in Canada compared with the synthetic control. These changes coincided with a $\$ 1,820$ million (95\% CI: $1,179.2$ to $2,464.1$ ) increase in US investment in the Canadian food and beverage sector, and a $\$ 5,258.3$ million (95\% CI: $4,894.8$ to $5,621.8$ ) rise in food and beverage imports from the US. This estimated rise in caloric intake corresponds to an average individual weight gain of $5.7 \mathrm{~kg}$ for women and $5.1 \mathrm{~kg}$ for men aged 40 and with low physical activity levels, or $2.5 \mathrm{~kg}$ for women and $2.2 \mathrm{~kg}$ for men aged 40 and who are very active.

Conclusion Our findings suggest that US FTA can substantially alter dietary behaviour by increasing caloric intake. FTA negotiations may be a critical window for shaping dietary behaviours to prevent overnutrition, obesity and related diseases.

\section{OP76 EVALUATING THE HEALTH IMPACTS OF RESTRICTIONS TO INCOME SUPPORT FOR LONE PARENTS: A NATURAL EXPERIMENT STUDY USING UNDERSTANDING SOCIETY}

SV Katikireddi*, O Molaodi, M Gibson, R Dundas, P Craig. MRC/CSO Social and Public Health Sciences Unit, University of Glasgow, Glasgow, UK

\subsection{6/jech-2017-SSMAbstracts.75}

Background Lone parents experience poorer health and socioeconomic disadvantage compared to coupled parents. The UK Government has restricted access to Income Support, the primary income replacement benefit for lone parents. Under Lone Parent Obligations (LPO), lone parents are transferred from Income Support to Jobseeker's Allowance once their youngest child reaches an age threshold (which has been reduced incrementally), and they must prove they are actively seeking work or face sanctions. We investigated the effects of introducing LPO on the health of lone mothers in the UK.

Methods We analysed 2009-2013 data from Understanding Society, a panel study representative of the UK general population. Our primary outcome was the mental health component of SF-12, with the physical health component and selfrated health assessed as secondary outcomes. We identified two intervention groups: lone parents newly exposed following the cut-off change from seven to five years and from ten to seven years. Each of these intervention groups were compared to two control groups: remaining unexposed (since the youngest child was below the age cut-off), and already exposed (since the youngest child was older than the existing age cut-off). We estimated a pooled LPO effect to increase precision. We conducted a difference-in-difference analysis using linear regression to estimate the 'intention to treat' causal effect, adjusting for maternal age, number of children and maternal education. Multiple imputation was used to address item missingness.

Results Our primary analysis included a total of 2257 participants. Mental health of lone parents consistently declined in intervention groups compared to control groups, whereas physical health and self-rated health showed little change. For lone parents with children aged 5-7 years who were newly exposed, the mental health score of SF-12 changed by -1.39 $(95 \% \mathrm{CI}-4.08,1.29)$ compared to those unexposed and -2.29 (95\%CI $-4.57,0.00)$ compared to those remaining exposed. Equivalent figures for the cut-off change from 10-7 years were $-2.45 \quad(95 \% \mathrm{CI}-5.48,0.57)$ and $-1.28 \quad(95 \% \mathrm{CI}$ $-4.00,1.45)$, while for the pooled effect were -2.13 (95\% CI $-4.17,-0.10)$ and $-2.21(95 \% \mathrm{CI}-4.13,-0.30)$. A complete case analysis and inclusion of males within the analytical sample led to similar results.

Discussion Increasing conditionality attached to the receipt of welfare benefits adversely impacted mental health of lone mothers but had no short-term impacts on physical health. Our study had limited statistical power and was only able to investigate short-term effects, but the pattern of findings was consistent across comparison groups. Planned extensions to LPO should be reconsidered.

\section{Neighbourhoods and communities}

\section{OP77 EVALUATING THE ASSET MODEL: FINDINGS FROM A RAPID REVIEW OF EVALUATION STRATEGIES}

1J South*, ${ }^{1}$ S Rippon, ${ }^{1} \mathrm{~K}$ Kinsella, ${ }^{1} \mathrm{AM}$ Bagnall. 'School of Health and Community Studies, Leeds Beckett University, Leeds, UK; ${ }^{2}$, Aligned Consulting Ltd, UK

\subsection{6/jech-2017-SSMAbstracts.76}

Background Ten years on from Morgan and Ziglio's call to revitalise the evidence base for public health through an asset model, there are still gaps in understanding about how best to evaluate asset-based approaches. The demand for better evidence poses some challenges as asset-based working emerges from radically different traditions from the mainstream deficit model in public health. This paper will present findings from a rapid evidence review on the measurement of asset-based approaches.

Aims The rapid review aimed to improve understanding of categories of measurement in the evaluation of asset-based approaches for health and wellbeing and to identify indicators and frameworks that can used in practice. The review was part of a project to develop an asset-based theory of change in health, care and wellbeing, funded by the Health Foundation.

Methods Rapid Evidence Assessment techniques were used to produce a map of evaluation approaches and measures. The search strategy used strings of common terms (eg Salutogensis, Asset Based Community Development) combined with synonyms of measurement/evaluation. Academic databases were searched from 2005 along with relevant websites. Additionally, the results of a previous search were screened and reference lists mined. Publications were included if they explicitly discussed the asset model and measurement. Data were then extracted across various fields including population, conceptual framework, measurement type and application. Findings were summarised in tables, with some additional thematic analysis.

Results 8689 publications were identified and 91 full text articles assessed for eligibility. In total, 33 publications were included in the review. Many of these flagged up the challenges of evaluation and the need for better outcome measurement. Reported research strategies varied considerably from the practical to the theoretical. Also the purpose, level and specificity of measurement ranged from validated indicators through to broad domain frameworks. Seven clusters were identified: Asset Based Community Development; Asset Mapping; Community-based evaluation; Conceptual frameworks for measurement; Resilience; Salutogensis; Other. One key theme was the importance of communities being involved in the assessment of assets. 
Conclusion The review provides an overview of research strategies and measures with application and purpose described. Producing a map of how asset-based approaches are evaluated and articulating key methodological differences helps researchers and practitioners select appropriate evaluation methods. There are a number of limitations, including the use of rapid review methods which may have missed other relevant evaluation approaches. Further methodological development is needed in this field and we welcome debate about ways to evaluate asset-based approaches.

\section{OP78 NEIGHBOURHOOD SOCIAL COHESION, ETHNICITY AND PHYSICAL ACTIVITY IN ADOLESCENTS: LONGITUDINAL EVIDENCE FROM THE ORIEL STUDY}

${ }^{1} \mathrm{~N}$ Berger*, 'DJ Lewis, ${ }^{2}$ EN Njagi, ${ }^{1} S$ Cummins. 'Social and Environmental Health Research, London School of Hygiene and Tropical Medicine, London, UK; ${ }^{2}$ Non-communicable Disease Epidemiology, London School of Hygiene and Tropical Medicine, London, UK

\subsection{6/jech-2017-SSMAbstracts.77}

Background Most adolescents do not achieve the recommended level of physical activity (PA) in the UK. Cultural norms - captured by ethnic identity - and social cohesion are aspects of the social environment that have the potential to influence health (behaviours). This study examines the relationships between social cohesion, ethnicity and three common types of PA in adolescents. The objectives are to test whether different types of PA have similar patterns of associations with social cohesion and ethnicity and to investigate confounding and interaction effects.

Methods We used longitudinal data from the Olympic Regeneration in East London (ORiEL) study. In 2012, 3088 adolescents aged 11-12 were recruited from 25 schools in four deprived and ethnically diverse boroughs of East London. Adolescents were followed-up in 2013 and 2014. Social cohesion was operationalised as trust in people living in the neighbourhood, measured on a four-point scale at wave 2. We grouped ethnic identities into eight categories. The outcomes were self-reported binary variables: walking to school, walking for leisure, and outdoor PA in the neighbourhood. We identified potential confounders. We fitted Generalised Estimated Equation models for each outcome, with a time trend and investigated potential interactions between the exposures and time. Final available case sample size was 4811 observations from 2157 adolescents.

Results We found evidence of associations between ethnicity and the PA outcomes. Adjustment for potential confounders (socioeconomic status, health and gender) did not attenuate the relationships. In the fully adjusted models, compared to White British, Black Africans had 1.49 higher odds of outdoor PA (95\% CI 1.06-2.07), Bangladeshis had 1.42 higher odds of walking to school (95\% CI 1.02-1.97) and all ethnic groups had lower odds of walking for leisure (estimated ORs varied between 0.37 and 0.71 ). While social cohesion was not associated with walking, there was evidence of a dose-response relationship with outdoor PA. A one-category increase in cohesion increasedthe odds of outdoor PA by 1.18 (95\% CI 1.071.30). We found no evidence of time*exposure interaction; exposures did not predict change in PA.

Conclusion This study contributes new findings to the evidence base on the social environment and PA. The ethnic diversity of the ORiEL study and its large sample size provided sufficient power to reveal how PA behaviours are patterned by ethnic groups. Further analyses will jointly model the three PA outcomes to better capture the dependency and associations between the exposures and the outcomes.

\section{OP79 INDIVIDUAL AND NEIGHBOURHOOD-LEVEL SOCIOECONOMIC FACTORS AND INCIDENCE OF TYPE 2 DIABETES IN OLDER AGE: RESULTS FROM A 14 YEAR FOLLOW-UP OF A COHORT OF OLDER BRITISH MEN}

${ }^{1}$ SE Ramsay, ${ }^{2} \mathrm{D}$ Roberts, ${ }^{3} \mathrm{AO}$ Papacosta, ${ }^{3} \mathrm{LT}$ Lennon, ${ }^{5} \mathrm{PH}$ Whincup, ${ }^{3} \mathrm{SG}$ Wannamethee* ${ }^{1}$ Institute of Health and Society, Newcastle University, Newcastle upon Tyne, UK; ${ }^{2}$ Whittington Health NHS Trust, Whittington Health NHS Trust, London, UK; ${ }^{3}$ Department of Primary Care and Population Health, UCL, London, UK; ${ }^{4}$ Population Health Research Institute, St George's, University of London, London, UK

\subsection{6/jech-2017-SSMAbstracts.78}

Background Current evidence linking socioeconomic factors to incident Type 2 Diabetes Mellitus (T2DM) in older populations is conflicting. We investigated the prospective association of individual socioeconomic position and neighbourhood-level socioeconomic deprivation with incident T2DM in older British men, and examined possible underlying factors.

Methods A socially-representative cohort of 3487 men, aged 60-79 years in 1998-2000, from 24 British towns was followed-up for 14 years for incident cases of T2DM. Individual socioeconomic position was based on social class derived from the longest-held occupation in middle-age, and was categorised into non-manual and manual groups. Neighbourhood-level socioeconomic deprivation was based on national Index of Multiple Deprivation (IMD) quintiles; a composite score of neighbourhood-level factors (income, employment, education, disability, crime, housing and living environment), with a higher score indicating greater deprivation. Follow-up on type 2 diabetes was obtained from reviews of general practitioner records and self-reported from questionnaires. Cox proportional hazards models were used to obtain hazard ratios (HR) and 95\% CI for incident diabetes according to social class and IMD quintiles. Prevalent cases of diabetes at baseline were excluded from the analyses.

Results During the follow-up of 14 years, there were 289 incident cases of T2DM (7.1 per 1000 person-years). Diabetes risk increased from higher to lower social class groups and from IMD quintile 1 (least deprived) to quintile 5 (most deprived) ( $\mathrm{P}$ for trend=0.001). Compared with non-manual social class groups, age-adjusted HR for manual groups was $1.58(95 \% \mathrm{CI} 1.24-2.01)$ - this was largely attenuated (1.38; 95\% CI 1.08-1.76) on adjustment for body mass index (BMI); adjustment for blood pressure, smoking, alcohol, physical activity, diet, medication and family history resulted in little attenuation while further adjustment for triglyceride levels attenuated the association. Compared with IMD quintile 1, the risk of incident T2DM was highest in IMD quintile 4 $(\mathrm{HR}=1.79$; 95\% CI 1.24-2.54). This largely attenuated on adjustment for BMI (HR=1.46; 95\% CI 1.02-2.10), and became non-significant after adjustment for lifestyle factors (smoking, alcohol, physical activity and diet).

Conclusion Manual social class and neighbourhood-level socioeconomic deprivation was associated with an increased risk of T2DM in older British men. For social class this was mostly explained by BMI and triglycerides. For neighbourhood-level socioeconomic deprivation it was largely explained by BMI and lifestyle factors. Our results support the need for public health initiatives specifically targeting obesity as a means 